\title{
KINESITHERAPY - A MAJOR METHOD OF CONSERVATIVE THERAPY FOR PATIENTS WITH FROZEN SHOULDER
}

\author{
Ilia Todorov \\ Clinic of Physical Therapy and Rehabilitation, University Hospital "St. Marina", \\ Medical University of Varna
}

\begin{abstract} tic approach. end.

\section{INTRODUCTION}

For most people frozen shoulder is a self-limiting condition of approximately $1-3$ years duration and it can be extremely painful and debilitating. People with the condition may struggle with basic daily activities and be worn down by sleep disturbance as a result of the pain (3).
\end{abstract}

PURPOSE: To review the scientific research about the incorporation and efficiency of the different kinesitherapy procedures for patients with frozen shoulder. To make conclusions and suggest a proper therapeu-

MATERIALS AND METHODS: All available sources, including literature and internet database have been examined. The latest scientific research articles have been represented in brief and summarized at the

RESULTS: According to the majority of the authors the kinesitherapy is a major method of conservative treatment that is recommended to be used in both primary and secondary care.

CONCLUSION: The low cost of the method together with the good clinical results are arguments in favor of the mass incorporation of the kinesitherapy in both primary and secondary care

Keywords: frozen shoulder, kinesitherapy, conservative treatment, combining methods

\footnotetext{
Address for correspondence:

Ilia Todorov Todorov, $M D$

Senior Assistant, Clinic of Physical Therapy and

Rehabilitation,

University Hospital "St. Marina"

1 Hristo Smirnenski Str, 9010 Varna

e-mail: ilkotodorov@gmail.com

tel: 052302851
}

Received: May 12, 2014

Accepted: September 10, 2014
As well as interfering with domestic and social activities, it can affect the ability to work. There may not be a complete resolution for all patients and there is variation across case series in the proportion of patients who do not regain full shoulder motion (4).

The pathology of the frozen shoulder involves different anatomical parts of the capsule of the glenohumeral joint. For the aggravated forms changes in the periarticular structures, mainly the muscles of the rotator cuff, are a common finding.

The conservative therapy of the disease usually comprises of physical procedures, kinesitherapy and drug therapy.

Concerning the application of corticosteroid injections, most of the authors have found a quick, but rapidly diminishing in terms of killing the pain effect. Most of them also announce a lack of increase in the limited range of motion. 
According to recent research physical therapy has a little better effectiveness than steroid injections. Because of the variety of the physical factors and different therapeutic combination options, there are still many therapies which were never researched. Exactly there may be hidden the opportunity of increasing the effectiveness of physical procedures by changing the therapeutic parameters and by combining with other factors.

Only a few of the methods of kinesitherapy have been researched in the recent years. The debate for the incorporation of physical therapy in treatment of frozen shoulder patients is still open.

In 1983 Rizk et al. compared in a research two interesting complexes of physical procedures (12). Group A comprised of 26 patients with 28 frozen shoulders that were administered with thermal therapy, kinesitherapy (Codman, pulley and wheel exercises and gentle, rhythmic stabilization type manipulation of the glenohumeral joint). Group B had 24 patients with 28 affected shoulders and the procedures conducted were TENS, pulley exercise and an interrupted traction with 2 hours duration $(15 \mathrm{~min}$. traction, $5 \mathrm{~min}$. pause). The average age of the patients was 56, and the symptoms of frozen shoulder were present 3 to 8 months before the research therapy started. The therapeutic combinations described were conducted for 8 weeks without interruption and evaluation of the accomplished results was performed every month for 6 consecutive months. During the first three weeks of the program group B progressed better than $\mathrm{A}$. The most significant improvement in both groups was observed in the first three weeks. As a final conclusion the authors declared a better effectiveness of the group B program. No statistical analysis were provided for that research.

Bulgen at al. in 1984 (1) conducted a randomized controlled clinical trial comparing the following therapeutic approaches: intraarticular steroid injection once per week for three weeks; 3 times per week manual mobilizations for 6 weeks; cryotherapy and PNF (proprioceptive neuromuscular facilitation) 3 times weekly for 6 weeks; no special therapy group. All patients were performing pendulum exercises for 2-3 minutes every hour during the day. Major inclusion criteria were shoulder pain lasting more than one month, sleep disturbances and incapability of ly- ing on the damaged side. Forty-two patients were admitted for the trial. Their average age was 55.8 years old, and the average duration of major symptoms before the trial start -4.8 months. Follow-up was performed once per week for the first 6 weeks, after that - once per month up to the $6^{\text {th }}$ month. The most significant improvement in all four groups has been observed in the first four weeks, and there was no significant long term superiority of any of the therapies used. The authors have proven a correlation between the higher patients' age and the bigger range of motion loss, excluding external rotation.

In 1985 Nicholson (10) compared the effectiveness of active kinesitherapy with manual joint mobilizations for 20 patients. The group with mobilizations achieved a bigger improvement in regaining internal rotation and abduction. One follow-up was conducted four weeks after the end of the therapy.

The first long-term follow-up trial was performed by Shaffer, Tibone and Kerlan (13) in 1992. They estimated objective and subjective results obtained from 62 patients with shoulder pain and limited range of motion from more than one month, abduction less than $100^{\circ}$ and decreased external rotation with more than $50 \%$. The average age was 52 years old and average duration of symptoms before the beginning of the treatment was 6 months.

The follow-up of the patients extended between 2 years and 6 months and 11 years and 9 months (average 7 years). All patients had conducted physical therapy in a hospital or home-designed stretch program at an earlier stage of the disease. Ten of the patients have had a manipulation of the glenohumeral joint under anesthesia and another two - arthroscopic intervention for a release of the capsule from the adhesions.

At the end of the trial the authors concluded the following:

* the average time for retrieval of the range of motion of the treated patients was 6 months (recovered up to minus $15^{\circ}$ of the standard);

* the average disease duration was 12 months;

* the pain has been gradually decreased and eventually eliminated for an average of 6 months;

* $31 \%$ of the patients were with moderate stiffness or moderate pain in the shoulder 
* 37 of the patients $(60 \%)$ in the research were with diminished range of motion in comparison with $0 \%$ in the control group (comprised of contralateral shoulders without any complains);

* only 7 patients (11\%) had minor functional limitations at the end of the follow-up

* - the patients with a significant limitation of the range of motion in all three anatomic plains were 13 times more often men than women $(\mathrm{p}>0.5)$;

* no correlation between the functional limitation and measured decrease in the range of motion was found;

* no correlation between the objective range of motion and the duration of the complains was found.

O'Kane at al. (11) studied the effect of an autostretch program on the patients' self-evaluation of the glenohumeral joint function. The patients assessed themselves with the questionnaire Simple Shoulder Test (12 questions) and the online questionnaire SF36 (36 questions). The authors reported that all the alterations in standards from SF-36 were eliminated after the treatment. Unclear about this research is the duration of the patients follow-up.

An year later Griggs et al. (5) studied the effect of a specific, including four directions, stretch home program. Seventy-five patients participated with an average age of 56. Periodically the authors conducted clinical assessment of pain, range of motion and function. All the patients completed the DASH questionnaire and the online SF-36. Patients were followed-up for an average of 22 months. Ninety percent declare the therapeutic results to be satisfactory and only $10 \%$ were not content. Male gender and diabetes were proven to be factors that worsen the final prognosis in cases of exacerbated symptoms. A significant improvement of the pain at rest and pain during motion was found ( $\mathrm{p}>0.001)$. As a whole, the results from the questionnaires were good even for patients with a smaller change in the range of moton, which proves their lower authenticity regarding the range of motion.

In 2000 Vermeulen et al. (14) in a scientific report described how they had applied physical therapy on 7 patients ( 4 men and 3 women), achieving an improvement in the range of motion and decrease of the pain. The therapy consisted of manual mobilizations twice a week for 3 consecutive months. Follow-up assessments of pain, joint mobility and function were conducted before and after the treatment, and later at the ninth month. An arthrographic evaluation of the joint volume was conducted to all patients (a solution of Haxabrix and Xylocaine 2\% in ratio 1:1 was injected in the joint until a feeling for a firm resistance was felt). The authors considered that the volume of the inserted in the joint space liquid corresponds with the joint volume. A decreased under $15 \mathrm{sm}^{3}$ volume in combination with an obliteration of bursa subscapularis and recessus axillaris at the arthrogram are typical findings for the adhesive capsulitis $(8,37)$. There was no statistical analysis of the research results. The research of Guler-Usal from 2004 (6) studied and examined the effect of two different physical therapy combinations - deep friction massage and mobilizations by Cyriax for the first group and a combination of centimeter waves and hot packages for the second. The author reports a supremacy of the Cyriax techniques, although the combination of endogenous and exogenous heat is rather controversial.

In 2006 again Vermeulen (15) et al. conducted a profound research based on the effect of different mobilizations for patients with frozen shoulder. A total of hundred patients participated, divided into two groups: group I treated with intensive, passive mobilizations reaching the end-range of motion in the glenohumeral joint; group II - gentle, low-amplitude passive mobilizations in the pain-free range of motion. All patients conducted 24 therapies (twice weekly for 12 consecutive weeks). Group I showed a statistically accredited greater improvement in all researched ranges of motion, the differences between the two groups being quite small.

In 2007 an interesting research was organized by Johnson et al. (7), comparing the effect of mobilizations with forward and backward shift, which are common techniques in the manual medicine complex for patients with frozen shoulder. Twenty patients were divided in two groups treated with $6 \mathrm{ul}$ trasound procedures, joint mobilizations and ergometer exercises for the upper extremities. The pain reduction in both groups was equal, but the group with backward shift mobilizations achieved a significantly greater improvement of the external rotation 
$(\mathrm{p}<0.001)$, the average range of improvement being $31.3^{\circ}$.

Another research from the same year (16) proves a supremacy regarding an increase of the range of motion from intensive, passive mobilizations reaching the end-range of motion and mobilizations with motion based on the Mulligan school (8, 9) compared with low-amplitude, pain-free passive mobilizations.

One of the latest researches of the methods of physical medicine is that of Cheing et al. (2) focusing on the effectiveness of combining interferent current (4-pole, $80-120 \mathrm{~Hz}$ ) with kinesitherapy home complex. The control group patients were only observed, which is a disadvantage. A significant improvement in pain and range of motion was reported at the fourth month follow-up.

\section{CONCLUSIONS}

As a summary most of the authors consider that:

* kinesitherapy has proven as the method with the highest efficiency regarding the improvement in the range of motion;

* the researches that upgrade it with other methods are only a few;

* almost $90 \%$ of the patients achieve not only a significant improvement in the range of motion, but also a considerable shortening of the disease duration

Major problems for the kinesitherapy are:

1. Acute pain in motion.

2. Incorrect conduction of the kinesitherapy.

3. Both lead to a worsening of the patient.

It shouldn't be forgotten, that one of the strongest opportunities of physical therapy is the possible combining and amplifying of the effects of the different physical factors. In that scientific direction a lot of research is about to commence and this combining of kinesitherapy and other types of physical therapy could make a shortening of the conservative therapy duration possible.

\section{REFERENCES}

1. Bulgen, D.Y., Binder, A.I., Hazleman, D. L., et

al. Frozen shoulder: prospective study with an evaluation of three treatment regiments. Ann Rheum Dis 1984;43:353-360.

2. Cheing, G.L.Y., So, E.M.L., Chao, C.Y.L. Effectiveness of electroacupuncture and interferential electrotherapy in the management of frozen shoulder. Journal of Rehabilitation Medicine, 2008;40:166-170.

3. Dawson, J., Shepperd, S., Carr, A. An overview of factors relevant to undertaking research and reviews on the effectiveness of treatment for frozen shoulder. Shoulder Elbow, 2010;2:232-7.

4. Dias, R., Cutts, S., Massoud, S. Frozen shoulder. BMJ 2005;331:1453-6.

5. Griggs, S. M., Ahn, A., Green, A. Idiopathic adhesive capsulitis: a prospective functional outcome study of non-operative treatment. J Bone Joint Surg Am, 2000;82(10):1398 - 1407.

6. Guler-Uysal, F., Kozanoglu, E. Comparison of the early response to two methods of rehabilitation in adhesive capsulitis, Swiss Medical Weekly, 2004, 134;353-358.

7. Johnson, A.J., Godges, J.J., Zimmerman, G.J., Ounanian, L.L.: The effect of anterior versus posterior glide joint mobilization on external rotation range of motion in patients with shoulder adhesive capsulitis. Journal Of Orthopaedic \& Sports Physical Therapy, 2007, 37, 3, 88-99.

8. Mulligan, B.R. Mobilisations with movement. J Manual Manipulative Ther, 1993;1:154-156.

9. Mulligan, B.R. Manual Therapy. "NAGS," "SNAGS," "MWMS," etc. 4th ed. Wellington, New Zealand: Plane View Services Ltd, 1999.

10. Nicholson G.G. The effects of passive joint mobilization on pain and hypomobility associated with adhesive capsulitis of the shoulder. J Orthop Sports Phys Ther, 1985;6:238-246.

11. O'Kane, J.W., Jackins, S., Sidles, J.A., et al. Simple home program for frozen shoulder to improve patient's assessment of shoulder function and health status. J Am Board Fam Pract, 1999;22(4):270-277.

12. Rizk, T.E., Christopher R.P., Pinals R.S., et al. Adhesive capsulitis: a new approach to its management. Arch Phys Med Rehabil, 1983;4:29-33.

13. Shaffer B., Tibone J.E., Kerlan R.K.: Frozen shoulder: a long-term follow-up. J Bone Joint Surg Am, 1992;74(5):738-746. 
14. Vermeulen, H.M, Rozing, P.M., Obermann, W.R., et al. Coparison of high-grade and low-grade mobilization techniques in the management of adhesive capsulitis of the shoulder: randomized controlled trial. Phys Ther, 2006;86:355-368.

15. Vermeulen H M, Obermann W R, Burger B J, et al.: End range mobilization techniques inadhesive capsulitis of the shoulder joint: a multiple subject case report. Phys Ther, 2000;80(12):1204 - 1213.

16. Yang, J-I., Chang, C-w., Chen, S-y., Wang, S-F., Lin, J-j. Mobilization techniques in subjects with frozen shoulder syndrome: Randomized multiple-treatment trial. Physical Therapy, 2007;87:1307-1315. 